\title{
Turning a low $Q$ fiber resonator into a high-sensitivity displacement sensor using slow light concepts
}

\author{
Mikel Bravo $^{\mathrm{a}, *}$, Xabier Angulo-Vinuesa ${ }^{\mathrm{b}}$, Sonia Martin-Lopez ${ }^{\mathrm{b}}$, Manuel Lopez-Amo ${ }^{\mathrm{a}}$ and Miguel \\ Gonzalez-Herraez ${ }^{\mathrm{c}}$ \\ ${ }^{a}$ Departamento de Ingeniería Eléctrica y Electrónica, Universidad Pública de Navarra, Pamplona \\ (Spain); \\ ${ }^{\mathrm{b}}$ Instituto de Óptica - Consejo Superior de Investigaciones Científicas, Madrid (Spain); \\ ${ }^{\mathrm{c}}$ Departamento de Electrónica - Escuela Politécnica, Universidad de Alcalá, Alcalá de Henares
} (Spain)

\begin{abstract}
High-Q resonators have been widely used for sensing purposes. High Q factors normally lead to sharp spectral peaks which accordingly provide a strong sensitivity in spectral interrogation methods. In this work we employ a low-Q ring resonator to develop a high sensitivity sub-micrometric resolution displacement sensor. We use the slow-light effects occurring close to the critical coupling regime to achieve high sensitivity in the device. By tuning the losses in the cavity close to the critical coupling, extremely high group delay variations can be achieved, which in turn introduce strong enhancements of the absorption of the structure. We first validate the concept using an Optical Vector Analyzer (OVA) and then we propose a simple functional scheme for achieving a low-cost interrogation of this kind of sensors.
\end{abstract}

Keywords: slow light, ring resonators, group delay, group index, Kerr effect.

\section{INTRODUCTION}

Slow light structures allow a large reduction of the group velocity of the light signals travelling through them, and, as a consequence, a strong confinement of the electromagnetic field and a great enhancement of light-matter interactions [1]. From the point of view of fiber sensors, the possibility of enhancing light-matter interactions may favor the development of more compact and sensitive devices. Some research effort has been devoted to understanding the exact enhancement values given by slow light in different light-matter interactions. For instance, the role of slow light in enhancing nonlinear effects has been theoretically investigated $[2,3]$. It has also been theoretically and experimentally proved that the extreme dispersion of slow light can lead to an enhancement in the spectral sensitivity of interferometers given by $n_{g}$ [4]. The role of slow light in enhancing gyroscope performance [5] and Beer-Lambert-Bouguer (BLB) absorption [6, 7] has also been theoretically and/or experimentally investigated. Generally speaking, light-matter interactions have been found to be only enhanced in structural slow light systems (e.g. coupled resonators, Bragg gratings, etc.). Material slow light systems (e.g. stimulated Brillouin/Raman scattering, parametric amplification, etc.) do not follow the same rules, the origin of this difference being that the electromagnetic energy velocity in material slow light does not depend on the group index [8]. From the fiber sensing point of view, it is therefore interesting to find structural slow light media, i.e. passive structures in which the group index could be tuned widely. Such a system could be considered as a platform for the development of slow-light assisted sensors. In this paper we demonstrate a wide range tuning of the group delay in a low-Q fiber-based ring resonator. This structure is shown to exhibit strong sensitivity enhancements when the resonator is tuned close to the critical coupling regime, where the slow light effects appear. Further to demonstrating the principle, we have also developed a functional interrogation scheme for displacement sensing with sub-micrometric resolution [9]. We believe that this structure could become interesting in future slow-light-assisted fiber sensors.

\section{THEORETICAL ANALYSIS}

We consider the structure depicted in Figure 1(a), i.e. the typical fiber ring resonator with a variable loss element inside. Our analysis of this structure is similar to the analysis done by Heebner et al. [10] for resonator-coupled waveguides. A transfer function can be found for the case of the lossy resonator: 


$$
\frac{E_{o}}{E_{i}}=\frac{\kappa \exp \left(\frac{i \omega n L}{c}\right)-a}{\exp \left(\frac{i \omega n L}{c}\right)-\kappa a}
$$

Where $\kappa$ and $a$ are, respectively, the coupling ratio and the attenuation in the resonator (in unitless form). As expected, this transfer function leads to resonances in the spectrum with a periodicity given by $c / n L$. These resonances exhibit steep dispersion slopes close to the center, leading to large group delays. We can theoretically evaluate the group delay in the resonances by finding the phase of expression (1), obtaining the derivative with respect to $\omega$, and evaluating the obtained expression at the resonant frequencies. The resulting expression is:

$$
\tau_{g}=\frac{n L}{c} \cdot \frac{a\left(1-\kappa^{2}\right)}{\kappa\left(a^{2}+1\right)-a\left(\kappa^{2}+1\right)}
$$

We can therefore see that the delay in the resonator can be expressed as the regular single-pass delay of the resonator $(n L / c)$ multiplied by a factor that may be varied continuously by changing only the attenuation and/or coupling ratio in the resonator. It can be easily shown that, when the losses exceed the coupling (undercoupling), negative group delays can be found at the resonances, while they remain positive in the opposite case (overcoupling). A discontinuity in function (2) is found for the critical coupling case $(a=\kappa)$, where the group delay function displays a vertical asymptote, taking values of $-\infty$ when $a \rightarrow \kappa^{-}$and $+\infty$ when $a \rightarrow \kappa^{+}$. The evolution of the group delay close to the critical coupling can be described by $\tau_{g} \propto(\kappa-a)^{-1}$. The qualitatively different behavior of both regimes is depicted in Fig. 2 .

We can now also evaluate the transmission coefficient of the resonator in the center of the resonance as a function of the attenuation and coupling coefficients. The power transmission coefficient can be written as:

$$
T=\left|\frac{a-\kappa}{\kappa a-1}\right|^{2}
$$

As it can be seen, in the transmission coefficient, the case $a=\kappa$ (critical coupling) corresponds to the case in which the transmission at the resonance center goes to zero. It is interesting now to evaluate the sensitivity of the log transmission to small attenuation/absorption changes in the ring.

$$
S=-\frac{d \ln T}{d a}=\frac{2}{\kappa-a}+\frac{2}{\kappa a-1}
$$

We can see two terms in this expression. The second one becomes extremely large when $\kappa a \rightarrow 1$, which corresponds to resonators with extremely high Q factors (typically used in sensing). In comparison, the first one show that the sensitivity can be very large working close to the critical coupling, were the slow light effects occur. In these conditions, the sensitivity can be seen to follow a similar dependence as the group delay $S \propto(\kappa-a)^{-1}$. It is interesting to stress that this enhancement is clearly not related to a high Q factor in the resonator, but to a slow light effect.

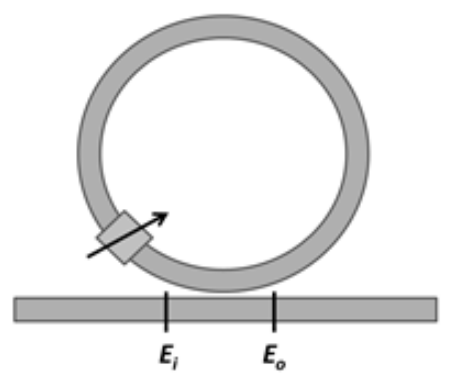

(a)
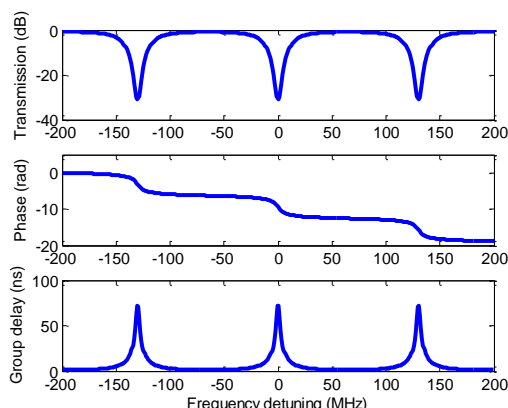

(b)

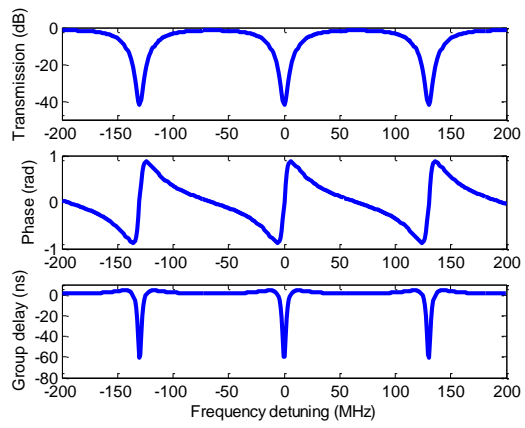

(c)

Fig. 1: (a) Fig.1: Fiber-based ring resonator considered. Light travels from left to right. (b) and (c) Phase, modulus and group delay response vs. frequency for different attenuation values within a 1.59 meter fiber resonator, $\kappa=0.7$ $(\sim 1.55 \mathrm{~dB})$ with (b) $1 \mathrm{~dB}$ attenuation in the loop; (c) $2 \mathrm{~dB}$ attenuation in the loop. 


\section{EXPERIMENTS}

We show here the development of a simple and high-sensitivity displacement sensor using the concepts shown before. Fig 2(a) shows the experimental setup used. A 70/30 fiber coupler is used to form the fiber ring. A couple of bending plates (BP) induce bending attenuation in the fiber as a function of the relative displacement. Fig 2(b) shows the attenuation induced as a function of the plate displacement. A polarization controller (PC) ensures correct polarization alignment within the resonator.

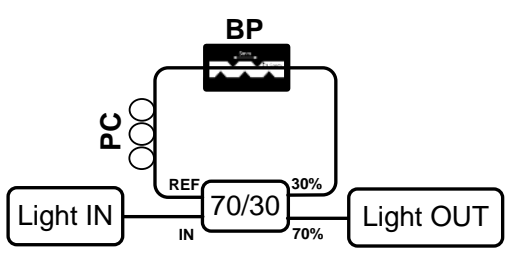

(a)

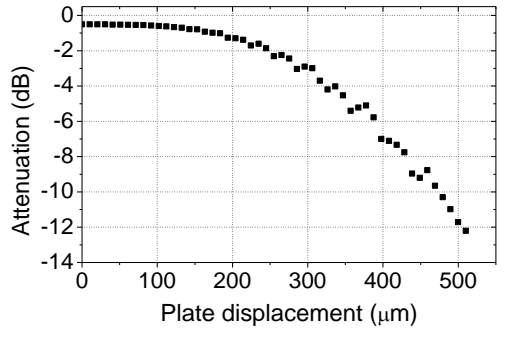

(b)

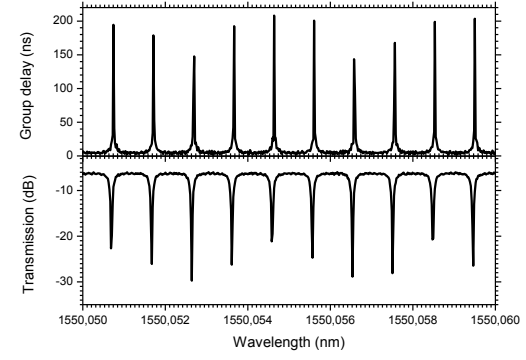

(c)

Fig. 2: (a) Experimental setup. (b) Attenuation induced by the plates as a function of the displacement. (c) Sample measurement of power transmission and group delay as a function of wavelength $(a \approx 1.7 \mathrm{~dB})$

In a first step, the characterization of the ring was done as a function of the attenuation in the ring induced by the bending plates. Both power transmission and group delay measurements were performed using an Optical Vector Analyzer (OVA). A sample measurement can be observed in Fig 2(c). Figure 3 depicts the (a) group delay and (b) transmission loss in the center of the resonances as a function of the attenuation induced by the plates studied with the OVA in [9]. The dots are experimental data and the blue lines correspond to the theoretical expectation obtained from the model developed in section 2 . We can see a good agreement between the results obtained with the experimental setup and the theoretical model. At critical coupling ratio $(\sim 1.55 \mathrm{~dB})$, the transition between positive group delay and negative group delay is visible (slow-fast-light switch).

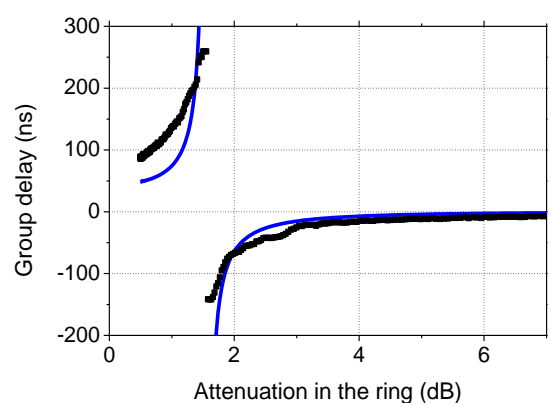

(a)

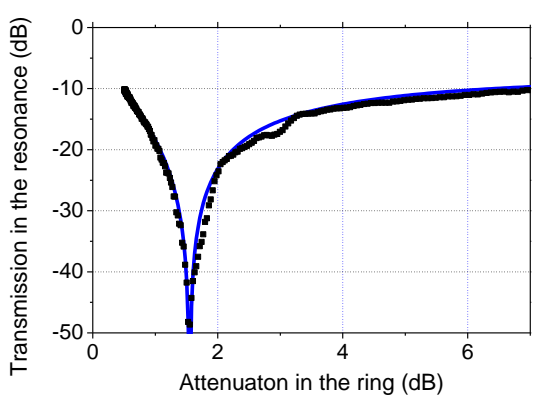

(b)

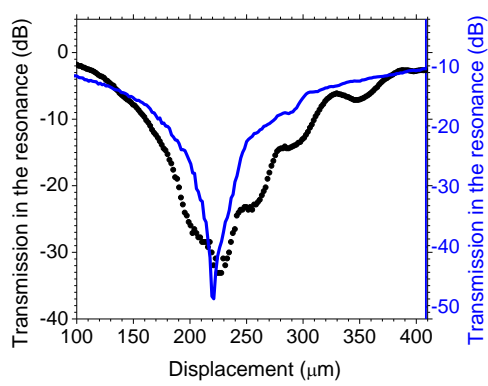

(c)

Fig. 3: (a) Measured peak group delay, (b) peak loss as a function of the attenuation induced in the ring and (c) power transmission as a function of the displacement. In (a) and (b), the dots correspond to measurements and the lines correspond to the theoretical expectation using the model shown in Section 2.

To validate the use of this setup as a real sensor, we interrogated the power transmission of the structure with an external-cavity tunable laser diode and a photodetector. Fine tuning of the diode wavelength is achieved by means of a piezo-electric actuator. Again, we measured the power transmission in the wavelength dips as a function of the displacement of the plates. The results can be seen in Figure 3(c), in comparison with the OVA results. Small oscillations 
due to cladding mode coupling effects are visible in the tunable laser measurement. Still, both OVA and the proposed setup results show a good agreement between them. As it can be seen, around the critical coupling regime, an enhanced sensitivity to displacement is achieved. In the particular case of the structure demonstrated in this work, the displacement sensitivity close to the critical coupling point exceeds $1 \mathrm{~dB} / \mu \mathrm{m}$. For comparison, this sensitivity value is more than two times the sensitivity achieved in [11] using the same bending plates in an interferometric structure.

\section{CONCLUSIONS}

We have theoretically and experimentally demonstrated a versatile all-fiber structure based on a lossy fiber ring resonator that can be used for sensing a variety of physical magnitudes (displacement, pressure, etc). The group delay and hence the loss sensitivity in the device is easily tuned by changing the attenuation and/or coupling ratio in the ring. Close to critical coupling, the setup can be made extremely sensitive to small variations in loss, in good agreement with the sharp group delay evolution in this region. The concept has been validated both theoretically and experimentally. It is important to highlight the development of a practical and more economic setup whose results are ingood agreement with the theoretical expectation. The sensitivity of any intensiometric fiber sensor can therefore be enhanced with this simple structure. This device could be a basic building block in new slow-light-assisted fiber sensors.

\section{ACKOWLEDGMENTS}

This work was supported by the European Research Council through Starting Grant U-FINE (Grant no. 307441), by the Spanish Ministry of Science and Innovation through projects TEC2012-37958-C02-01, TEC2010-20224-C02-01, Innocampus, and the Comunidad de Madrid through project FACTOTEM-2. Sonia Martin-Lopez acknowledges funding from the Spanish Ministry of Science and Innovation through a "Juan de la Cierva" contract.

\section{REFERENCES}

[1] J. B. Khurgin and R. S. Tucker, "Slow light: Science and applications," CRC Press, Boca Raton, 2009.

[2] M. Soljacic, S. G. Johnson, S. Fan, M. Ibanescu, E. Ippen, and J. D. Joannopoulos, "Photonic-crystal slow-light enhancement of nonlinear phase sensitivity,” J. Opt. Soc. Am. B 19, 2052, 2002.

[3] C. Monat, B. Corcoran, M. E. Heidari, C. Grillet, B. J. Eggleton, T. P. White, L. O’Faolain and T. F. Krauss, "Slow light enhancement of nonlinear effects in silicon engineered photonic crystal waveguides," Opt. Express 17, 2944-2953 (2008).

[4] Z. Shi, R. W. Boyd, D. J. Gauthier and C. C. Dudley, "Enhancing the spectral sensitivity of interferometers using slowlight media," Opt. Lett. 32, 915-917 (2007).

[5] M. Terrel, M. J. F. Digonnet and S. Fan, "Performance comparison of slow-light coupled-resonator optical gyroscopes," Laser \& Photon. Rev. 3, 452-465 (2009).

[6] N. A. Mortensen and S. Xiao, "Slow-light enhancement of Beer-Lambert-Bouguer absorption," Appl. Phys. Lett. 90, 141108 (2007).

[7] S. Chin, I. Dicaire, J. Beugnot, S. Foaleng-Mafang, M. Gonzalez-Herraez, and L. Thévenaz, "Material Slow Light Does Not Enhance Beer-Lambert Absorption," in Slow and Fast Light, OSA Technical Digest (CD) (Optical Society of America), paper SMA3 (2009)

[8] M. Santagiustina, "Electromagnetic Energy Velocity in Slow Light," in Slow and Fast Light, OSA Technical Digest (CD) (Optical Society of America), paper SLTuB5 (2011)

[9] M. Bravo, X. Angulo-Vinuesa, S. Martin-Lopez, M. Lopez-Amo, and M. Gonzalez-Herraez, "Versatile all-fiber slowlight assisted sensor," Proc. SPIE Photonic West, 8636-20 (2013)

[10] J. Heebner and R. W. Boyd, "Slow and fast light in resonator-coupled waveguides," J. Mod. Optics 49 2629-2636, 2002.

[11] M. Bravo, A. M. R. Pinto, M. Lopez-Amo, J. Kobelke, and K. Schuster, "High precision micro-displacement fiber sensor through a suspended-core Sagnac interferometer," Opt. Lett. 37, 202-204 (2012) 